\title{
Spin-transfer torque in magnetic tunnel junctions: Scattering theory
}

\author{
Jiang Xiao and Gerrit E. W. Bauer \\ Kavli Institute of NanoScience, Delft University of Technology, 2628 CJ Delft, The Netherlands
}

Arne Brataas

Department of Physics, Norwegian University of Science and Technology, NO-7491 Trondheim, Norway

(Received 29 March 2008; revised manuscript received 15 May 2008; published 12 June 2008)

\begin{abstract}
We study the bias-dependent spin-transfer torque in magnetic tunnel junctions in the Stoner model by scattering theory. We show that the in-plane (Slonczewski type) torque vanishes and subsequently reverses its direction when the bias voltage becomes larger or the barrier wider than material and device-dependent critical values. We are able to reproduce the magnitude and the bias dependence of measured in-plane and out-of-plane torques using realistic parameters. The condition for the vanishing torque is summarized by a phase diagram depending on the applied bias and barrier width, which is explained in terms of an interface spin polarization and the electron focusing by the barrier. Quantum size effects in the spin-transfer torque are predicted as a function of the thickness of a normal-metal layer inserted between the ferromagnet and tunnel barrier.
\end{abstract}

DOI: 10.1103/PhysRevB.77.224419

PACS number(s): 73.63.-b, 85.75.-d, 72.25.-b, 73.40.Gk

\section{INTRODUCTION}

Magnetic tunnel junctions (MTJ) are layered structures in which an insulating tunnel barrier $(I)$ separates two ferromagnetic layers $(F) .,{ }^{1,2}$ The interplay between electronic currents and an order-parameter difference, i.e., magnetizations rotated away from the equilibrium configurations, is the magnetic equivalent to the Josephson effect in superconductivity. The MTJ with a thin normal-metal insertion layer is the only magnetoelectronic structure that shows quantum size effects on electron transport. ${ }^{3}$ MTJs based on epitaxial $\mathrm{MgO}$ barriers ${ }^{4,5}$ are used in the magnetic random-access memory (MRAM) devices that are operated by the spintransfer torque. ${ }^{6,7}$ MTJs have been studied vigorously, initially focusing on the tunnel magnetoresistance (TMR). ${ }^{3,8-10}$ More recently, the focus shifted to spin-transfer torque and current-induced magnetization switching. ${ }^{11-24}$ On the theoretical front, spin-transfer effects have been studied extensively in metallic spin valve structures based on various models. ${ }^{25-30}$ For tunneling structures, such studies are still relatively scarce. ${ }^{15,17-20}$

Here we report a model study of the spin-transfer torque in magnetic tunnel junctions. Since the ferromagnets are separated by tunnel barriers, we cannot use theories existing for metallic structures that are mostly based on semiclassical methods..$^{25,31}$ Instead, we chose a fully quantum-mechanical treatment of transport through the tunnel barrier by scattering theory. The high quality of $\mathrm{MgO}$ tunnel junctions and the prominence of quantum oscillations observed in ferromagnetic-normal metal-insulator-ferromagnetic (FNIF) structures (even for alumina barriers) provide the motivation to concentrate on ballistic structures in which the transverse Bloch vector is conserved during transport. We qualitatively (and even quantitatively) confirm the results in Refs. 15, 19, and 20. However, our model is simpler and physically more transparent than the tight-binding method used in Ref. 15 and the numerical studies of Refs. 19 and 20. We are able to reproduce simultaneously both the in-plane and out-of-plane torque experimental data using realistic material and device parameters, in contrast to a fit based on the tight-binding model. ${ }^{22}$ We also show finite zero-bias out-of-plane torque for asymmetric structures. Scattering theory enables us to distill a clear physical picture of the peculiarities of the spintransfer torque in MTJs, which allows us to understand why and when the torque goes to zero. The torque zero-crossing condition can be summarized by a phase diagram spanned by the applied bias and barrier width parameters. With our approach we can go beyond the ferromagnetic-insulatorferromagnetic (FIF) MTJ and study the effect of a normalmetal insertion (FINF structures). Quantum size oscillations in the torque are predicted as a function of the thickness of the $N$ insertion layer.

This paper is organized as follows: Section II introduces the FIF and FNIF structures and the scattering theory. In Sec. III, approximations are introduced in order to derive analytic expressions. Section IV presents our main results. Section V compares our model with experimental results. Sections VI and VII contain a brief discussion and summary, respectively. Two appendices are attached at the end.

\section{STRUCTURE AND METHOD}

We consider multilayers as shown in Fig. 1(a) in which two semi-infinite $F$ leads $[F(L)$ and $F(R)]$ are connected by an insulating layer $(I)$ of width $d$ and a nonmagnetic metal layer $(N)$ of width $a$. The magnetization direction of $F(L) / F(R), \mathbf{m}_{1} / \mathbf{m}_{2}\left(\left|\mathbf{m}_{1}\right|=\left|\mathbf{m}_{2}\right|=1\right)$, is treated as fixed/free. This structure reduces to a conventional FIF MTJ when $a$ $=0$.

Let $A, B, C, D, C^{\prime}, D^{\prime}, E$, and $F$ be the spin-dependent amplitudes $A^{\dagger}=\left(A_{\uparrow}^{\dagger}, A^{\dagger}\right)$ of flux-normalized spinor wave functions at specific points. The scattering states can be expressed in terms of two incoming waves $A$ and $F$, such as:

$$
C^{\prime}=\hat{s}_{C^{\prime} A} A+\hat{s}_{C^{\prime} F} F,
$$

where $\hat{s}_{C^{\prime} A}$ and $\hat{s}_{C^{\prime} F}$ are $2 \times 2$ matrices in spin space that can be constructed by concatenating the scattering matrices of region $S_{1,2}$ and of the insulating layer bulk (see Fig. 1). To 


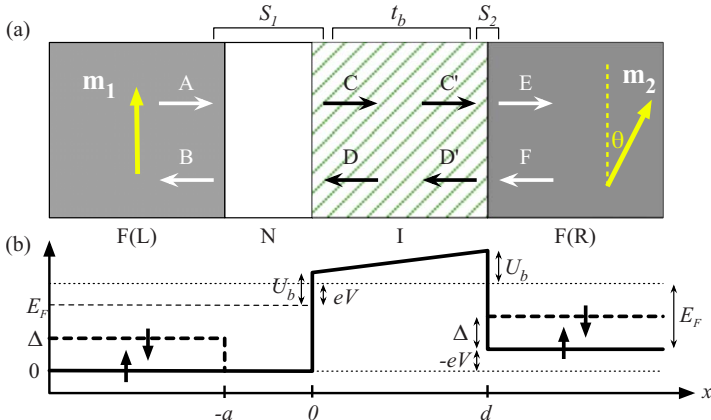

FIG. 1. (Color online) (a) FNIF heterostructure, in which $S_{1,2}$ indicate two different interface scattering regions; (b) The potential profiles (at positive bias) for majority and minority electron spins in $F$ are shown by solid and dashed lines, respectively. The exchange splitting is $\Delta$ and the tunnel barrier has height $U_{b}$ relative to the Fermi energy $E_{F}$. The applied bias $V$ pictured in (b) corresponds to a net electron (particle) flow from right to left.

leading order of the transmission $\left(t_{b}\right)$ through the bulk I (similar expansions hold for $\hat{s}_{D^{\prime} A}$ and $\hat{s}_{D^{\prime} F}$ as well):

$$
\begin{gathered}
\hat{s}_{C^{\prime} A}=\left[\left(1-r_{b}^{\prime} \hat{r}_{2}\right)^{-1} t_{b}\left(1-\hat{r}_{1}^{\prime} r_{b}\right)^{-1}\right] \hat{t}_{1}, \\
\hat{s}_{C^{\prime} F}=\left[\left(1-r_{b}^{\prime} \hat{r}_{2}\right)^{-1} r_{b}^{\prime}+t_{b}\left(1-\hat{r}_{1}^{\prime} r_{b}\right)^{-1} \hat{r}_{1}^{\prime} t_{b}^{\prime}\left(1-\hat{r}_{2} r_{b}^{\prime}\right)^{-1}\right] \hat{t}_{2}^{\prime},
\end{gathered}
$$

where $\hat{t}_{1,2} / \hat{r}_{1,2}$ are the $2 \times 2$ transmission/reflection matrices for $S_{1,2}$ (see Fig. 1) and $t_{b} / r_{b}$ are the spin-independent transmission/reflection coefficient for the insulating bulk material that are proportional to the unit matrix in spin space and therefore without hat. The primed and unprimed versions indicate scattering of electrons impinging from the left and right, respectively. The reflection coefficient $r_{b}$ is due to the impurity scattering inside the bulk insulator, which, as $t_{b}$, contains an exponential decay factor representing evanescent states in $I$. For this reason the magnitude of $r_{b}$ is comparable to or much smaller than that of $t_{b}$ depending on the density of the impurities. All scattering matrices are matrices in $\mathbf{k}$ space defined by the propagating states of left and right leads in the energy window available for transport, labeled by their transverse wave vectors in the leads: $\mathbf{q}, \mathbf{q}^{\prime}$ (the band index is suppressed) at a given energy.

An applied bias voltage $V$ drives a (conserved) charge current $J_{c}$ and (spatially dependent) spin current $\mathbf{J}_{s}$ through the device. At zero temperature, the charge current reads,

$$
\begin{gathered}
J_{c}=\frac{1}{(2 \pi)^{3}} \int d E \sum_{\mathbf{q}, \mathbf{q}^{\prime}} j_{c}\left(\mathbf{q}, \mathbf{q}^{\prime}\right), \\
j_{c}=\frac{4 e}{\hbar} \operatorname{Tr}_{\sigma}\left[\operatorname{Im}\left(\hat{s}_{E A^{\prime}} \hat{s}_{E A}^{\dagger} f_{L}-\hat{s}_{E F} \hat{s}_{E F}^{\dagger} f_{R}\right)\right] .
\end{gathered}
$$

where $\operatorname{Tr}_{\sigma}[\cdots]$ denotes the spin trace and the summation is over all the transverse modes at energy $E . f_{L}=f_{L}(E)$ and $f_{R}$ $=f_{R}(E+e V)$ are (zero temperature) Fermi-Dirac electron distribution functions in the left and right reservoirs. We are therefore disregarding any spin accumulation in the ferro- magnet, which is valid for tunnel junctions of current interest in which the spin-flip rate in the ferromagnet is larger than the tunnel rate. The scattering matrices depend on $V$ by the bias-induced potential profile. The spin current, or the angular-momentum current, at the left side of the $I / F(R)$ interface (within $I$ ) reads

$$
\begin{gathered}
\mathbf{J}_{\mathrm{s}}=\frac{1}{(2 \pi)^{3}} \int d E \sum_{\mathbf{q}, \mathbf{q}^{\prime}} \mathbf{j}_{\mathrm{s}}\left(E, \mathbf{q}, \mathbf{q}^{\prime}\right), \\
\mathbf{j}_{s}=2 \operatorname{Tr}_{\sigma}\left[\hat{\boldsymbol{\sigma}} \operatorname{Im}\left(\hat{s}_{C^{\prime} A} \hat{s}_{D^{\prime} A}^{\dagger} f_{L}-\hat{s}_{C^{\prime} F} \hat{s}_{D^{\prime} F}^{\dagger} f_{R}\right)\right] .
\end{gathered}
$$

Since the spin current deep in the ferromagnetic (FM) lead is longitudinal to the magnetization, the torque $\mathbf{N}$ acting on $F(R)$ is equal to the transverse component of the incoming spin current that is absorbed at the interface: $6,25,26,28$

$$
\mathbf{N}=\mathbf{J}_{s}-\left(\mathbf{J}_{s} \cdot \mathbf{m}_{2}\right) \mathbf{m}_{2}=\mathbf{N}_{\|}+\mathbf{N}_{\perp},
$$

with the in-plane (Slonczewski) torque $\mathbf{N}_{\|} \propto \mathbf{m}_{2} \times\left(\mathbf{m}_{1} \times \mathbf{m}_{2}\right)$ and out-of-plane (fieldlike) torque $\mathbf{N}_{\perp} \propto \mathbf{m}_{1} \times \mathbf{m}_{2}$. Similarly $\mathbf{n}=\mathbf{j}_{s}-\left(\mathbf{j}_{s} \cdot \mathbf{m}_{2}\right) \mathbf{m}_{2}=\mathbf{n}_{\|}+\mathbf{n}_{\perp}$.

At low bias, the nonequilibrium part of the spin current is proportional to the bias voltage $\mathbf{J}_{s}-\mathbf{J}_{s}^{0}=\mathbf{G}_{s} V$, where $\mathbf{J}_{s}^{0}$ is the equilibrium spin current that is related to interlayer exchange coupling at equilibrium and $\mathbf{G}_{s}$ is the spin conductance:

$$
\begin{gathered}
\mathbf{G}_{s}=\frac{1}{(2 \pi)^{2}} \sum_{\mathbf{q}, \mathbf{q}^{\prime}}^{E=E_{F}} \mathbf{g}_{s}\left(\mathbf{k}, \mathbf{k}^{\prime}\right), \\
\mathbf{g}_{s}=\frac{e}{\pi} \operatorname{Tr}_{\sigma}\left[\hat{\boldsymbol{\sigma}} \operatorname{Im}\left(\hat{s}_{C^{\prime} A \hat{S}_{D^{\prime} A}^{\dagger}}+\hat{s}_{C^{\prime} F^{\hat{S}^{\prime}}}^{\dagger}{ }_{D^{\prime} F}\right)\right],
\end{gathered}
$$

where the scattering matrices are evaluated at zero bias $V$ $=0$ and the summation is over transport channels at the Fermi energy. We define the linear-response torkance ${ }^{30} \mathbf{T}$ $=\mathbf{N} / V, \quad$ and $\quad \mathbf{T}=\mathbf{G}_{s}-\left(\mathbf{G}_{s} \cdot \mathbf{m}_{2}\right) \mathbf{m}_{2}=\mathbf{T}_{\|}+\mathbf{T}_{\perp} \quad$ and $\quad \boldsymbol{\tau}=\mathbf{g}_{s}$ $-\left(\mathbf{g}_{s} \cdot \mathbf{m}_{2}\right) \mathbf{m}_{2}=\boldsymbol{\tau}_{\|}+\boldsymbol{\tau}_{\perp}$.

\section{APPROXIMATIONS}

We assume in the following that the spin is conserved during the scattering. Then $\hat{t}_{i}$ for $S_{i}\left(i=1,2\right.$, similar for $\left.\hat{r}_{i}\right)$ is diagonal when choosing $\mathbf{m}_{i}$ as spin-quantization axis: Expanded in Pauli matrices $\hat{\boldsymbol{\sigma}}=\left(\hat{\sigma}_{x}, \hat{\sigma}_{y}, \hat{\sigma}_{z}\right), \hat{t}_{i}=t_{i}^{+}+t_{i}^{-} \hat{\boldsymbol{\sigma}} \cdot \mathbf{m}_{i}$, with $t_{i}^{ \pm}=\left(t_{i}^{\uparrow} \pm t_{i}^{\downarrow}\right) / 2 . t_{i}^{\sigma}(\sigma=\uparrow, \downarrow)$ is the transmission amplitude for spin $\sigma$ for spin-quantization axes $\mathbf{m}_{i}$ through the scattering region $S_{i}$. In the absence of impurities $\left(r_{b}=0\right)$ and to leading order of $t_{b}$ :

$$
\begin{gathered}
\hat{s}_{C^{\prime} A}=t_{b}\left(t_{1}^{+}+t_{1}^{-} \hat{\boldsymbol{\sigma}} \cdot \mathbf{m}_{1}\right), \\
\hat{s}_{D^{\prime} A}=\left(r_{2}^{+} t_{b} t_{1}^{+}+r_{2}^{-} t_{b} t_{1}^{-} \mathbf{m}_{1} \cdot \mathbf{m}_{2}\right)+\hat{\boldsymbol{\sigma}} \cdot\left(r_{2}^{-} t_{b} t_{1}^{+} \mathbf{m}_{1}+r_{2}^{+} t_{b} t_{1}^{-} \mathbf{m}_{2}\right. \\
\left.-i r_{2}^{-} t_{b} t_{1}^{-} \mathbf{m}_{1} \times \mathbf{m}_{2}\right),
\end{gathered}
$$

Similar expansions hold for $\hat{s}_{C^{\prime} F}$ and $\hat{s}_{D^{\prime} F}$.

Next, we adopt the free-electron approximation tailored for transition-metal ferromagnets. ${ }^{8}$ We assume spherical Fermi surfaces for spin-up and spin-down electrons [in both 
$F(L)$ and $F(R)]$ with Fermi wave vectors $k_{F}^{\uparrow}=\sqrt{2 m E_{F} / \hbar^{2}}$ and $k_{F}^{\downarrow}=\sqrt{2 m\left(E_{F}-\Delta\right) / \hbar^{2}}$, with an effective electron mass $m$ in $F$. Electrons in $N$ are assumed to be ideally matched with the majority electrons in $F\left(k_{F}=k_{F}^{\uparrow}\right)$. The effective electron mass in the tunnel barrier is assumed to be $m_{b}=\beta m$. Figure 1(b) shows the adopted potential profile with barrier height $U_{b}$. We assume an applied potential bias that is smaller than the barrier height $|e V|<U_{b}$ that fully drops over the tunnel barrier. Positive bias corresponds to charge current flow from left to right and electron particle flow from right to left. As in Refs. 15, 18, and 19, we assume that energy and transverse wave vector $\mathbf{q}$ are conserved, thus disregard any impurity/ interface roughness scattering. All scattering matrices then become diagonal in $\mathbf{k}$ space.

In the free-electron model, the flux-normalized wave functions in $N$ and $F$ are:

$$
\psi_{ \pm}^{N}=\frac{e^{ \pm i k_{x} x}}{\sqrt{k_{x}}} \text { in } N \text { and } \psi_{ \pm}^{\sigma}=\frac{e^{ \pm i k_{x}^{\sigma} x}}{\sqrt{k_{x}^{\sigma}}} \text { in } F,
$$

Using the WKB approximation, ${ }^{18}$ the wave function in the tunneling barrier is

$$
\psi_{ \pm}^{b}=\frac{e^{ \pm \int_{0}^{x} \kappa(w) d w}}{\sqrt{ \pm i \kappa(x)}} \text { in } I
$$

with

$$
\kappa(x)=\sqrt{\frac{2 m_{b}}{\hbar^{2}}\left(U_{b}+E_{F}-E-e V \frac{x}{d}\right)+q^{2}},
$$

where $E$ is the energy of the electron. The WKB approximation is valid when the potential profile varies slowly in space within the tunneling barrier, i.e., $\kappa^{\prime}(x) \ll \kappa^{2}(x)$. The transmission coefficient through $I$ reads $t_{b}=\exp \left[-\int_{0}^{d} \kappa(w) d w\right]$.

For finite bias, from Eqs. (4), (7a), and (7b),

$$
\begin{gathered}
\mathbf{n}_{\|}=t_{b}^{2} T_{1}^{-} T_{2}^{+}\left(f_{L}-f_{R}\right) \mathbf{m}_{2} \times\left(\mathbf{m}_{1} \times \mathbf{m}_{2}\right), \\
\mathbf{n}_{\perp}=2 t_{b}^{2} \operatorname{Re}\left(T_{1}^{-} r_{2}^{-} f_{L}+T_{2}^{-} r_{1}^{\prime-} f_{R}\right) \mathbf{m}_{1} \times \mathbf{m}_{2}, \\
\mathbf{n}_{\perp}^{0}=2 t_{b}^{2} \operatorname{Re}\left(T_{1} r_{2}^{-}+T_{2} r_{1}^{\prime-}\right) f_{0} \mathbf{m}_{1} \times \mathbf{m}_{2},
\end{gathered}
$$

where $T_{i}^{+}=\left|t_{i}^{\uparrow}\right|^{2}+\left|t_{i}^{\downarrow}\right|^{2}$ is the average transmission probability for scattering region $S_{i}, T_{i}^{-}=p_{i} T_{i}^{+}=\left|t_{i}^{\uparrow}\right|^{2}-\left|t_{i}^{\downarrow}\right|^{2}$ with polarization $p_{i}=T_{i}^{-} / T_{i}^{+}$, and $f_{0}$ is the equilibrium distribution function at zero bias. $\mathbf{n}_{\perp}$ in Eq. (11b) includes both the equilibrium and nonequilibrium contribution to the out-of-plane torque. The former $\left[\mathbf{n}_{\perp}^{0}\right.$ in Eq. (11c)] is related with the nonlocal interlayer exchange coupling. ${ }^{32}$ The nonequilibrium contribution is therefore $\mathbf{n}_{\perp}^{\prime}=\mathbf{n}_{\perp}-\mathbf{n}_{\perp}^{0}$. The optical theorem $2 \operatorname{Im}\left(r_{1,2}^{ \pm}\right)=T_{1,2}^{ \pm}$(see Appendix $\mathrm{A}$ ) is used in the derivation of Eq. (11a) to get rid of all internal reflection in I. In Eq. (11a), we observe that the in-plane torque is caused by the polarization of the current at the left interface that is expressed by $T_{1}$. The subsequent absorption of the spin current by the second magnet is governed by the geometrical projection expressed by the vector product and the total transparency of the second interface $T_{2}^{+}$. It follows from Eq. (11b) that the out out-of-plane torque has a very different origin. It does not depend directly on the difference of the electron distributions on both sides of the junctions, but consists of two independent contributions from both reservoirs. Each contribution consists of the spin polarization of the first interface, but is sensitive to the phase of the reflection coefficient of the second interface. The out-of-plane torque can be interpreted as the net spin created at one interface that while reflected at the second interface briefly precesses in the exchange field of the second ferromagnet.

With vanishing bias, $t_{b}=\exp (-\kappa d)$ with $\kappa$ $=\sqrt{2 m_{b} U_{b} / \hbar^{2}+q^{2}}$. By Eqs. (6), (7a), and (7b),

$$
\boldsymbol{\tau}=\boldsymbol{\tau}_{\|}=\frac{e}{2 \pi} e^{-2 \kappa d} T_{1} T_{2}^{+} \mathbf{m}_{2} \times\left(\mathbf{m}_{1} \times \mathbf{m}_{2}\right),
$$

and $\boldsymbol{\tau}_{\perp}=0$. For reference, the conductance within the same theoretical framework is given by: ${ }^{33}$

$$
g_{\mathrm{c}}=\frac{e^{2}}{2 h} e^{-2 \kappa d}\left(T_{1}^{+} T_{2}^{+}+T_{1}^{-} T_{2}^{-} \mathbf{m}_{1} \cdot \mathbf{m}_{2}\right) .
$$

The vector product $\left|\mathbf{m}_{2} \times\left(\mathbf{m}_{1} \times \mathbf{m}_{2}\right)\right|=\sin \theta$ in Eq. (12) and $\mathbf{m}_{1} \cdot \mathbf{m}_{2}=\cos \theta$ in Eq. (13), leading to the well-known geometrical dependence of the angular transport properties of tunnel junctions. ${ }^{6}$ The vanishing of the out-of-plane torque $\boldsymbol{\tau}_{\perp}=0$, is a rather general result that holds for symmetric tunneling junctions and spin valves in the linear-response regime. ${ }^{15}$ We consider a symmetric system with an applied voltage $-V / 2$ to the left and a voltage $V / 2$ to the right reservoir. To the second order in the bias voltage, the spin current in the spacer between the ferromagnets can be expanded as

$$
\begin{aligned}
\mathbf{I}_{s}= & {\left[A_{1} \mathbf{m}_{1}+B_{1} \mathbf{m}_{2}+C_{1} \mathbf{m}_{1} \times \mathbf{m}_{2}\right] V } \\
& +\left[A_{2} \mathbf{m}_{1}+B_{2} \mathbf{m}_{2}+C_{2} \mathbf{m}_{1} \times \mathbf{m}_{2}\right] V^{2} .
\end{aligned}
$$

When applying the mirror operation left $\leftrightarrow$ right $(1 \leftrightarrow 2$, $\left.-V / 2 \leftrightarrow V / 2, \mathbf{I}_{s} \leftrightarrow-\mathbf{I}_{S}\right)$ symmetry requires that

$$
\begin{aligned}
-\mathbf{I}_{s}= & {\left[A_{1} \mathbf{m}_{2}+B_{1} \mathbf{m}_{1}+C_{1} \mathbf{m}_{2} \times \mathbf{m}_{1}\right](-V) } \\
& +\left[A_{2} \mathbf{m}_{2}+B_{2} \mathbf{m}_{1}+C_{2} \mathbf{m}_{2} \times \mathbf{m}_{1}\right](-V)^{2},
\end{aligned}
$$

which should be identical to Eq. (14). Therefore $A_{1}$ $=B_{1}, C_{1}=0, A_{2}=-B_{2}$, whereas $C_{2}$ is not restricted. Then the torque on $\mathbf{m}_{2}$ is

$$
\begin{aligned}
\mathbf{N}= & \mathbf{m}_{2} \times\left(\mathbf{I}_{s} \times \mathbf{m}_{2}\right)=\left[A_{1} V-A_{2} V^{2}\right] \mathbf{m}_{2} \times\left(\mathbf{m}_{1} \times \mathbf{m}_{2}\right) \\
& +C_{2} V^{2} \mathbf{m}_{1} \times \mathbf{m}_{2} .
\end{aligned}
$$

This proves that, for symmetric systems, the out-of-plane torkance $\left(\propto 2 C_{2} V\right)$ vanishes at $V=0$. It also shows that beyond linear response, there are quadratic (in bias) contributions to both the in-plane and out-of-plane torques. The argument does not hold for asymmetric tunneling systems. An experimental zero-bias out-of-plane torkance should therefore provide interesting information on MTJ asymmetries.

\section{RESULTS}

In this section, we discuss three different structures: (a) A symmetric FIF magnetic tunneling junction, (b) an asymmetric FIF structure in which the left and right FM layers have 

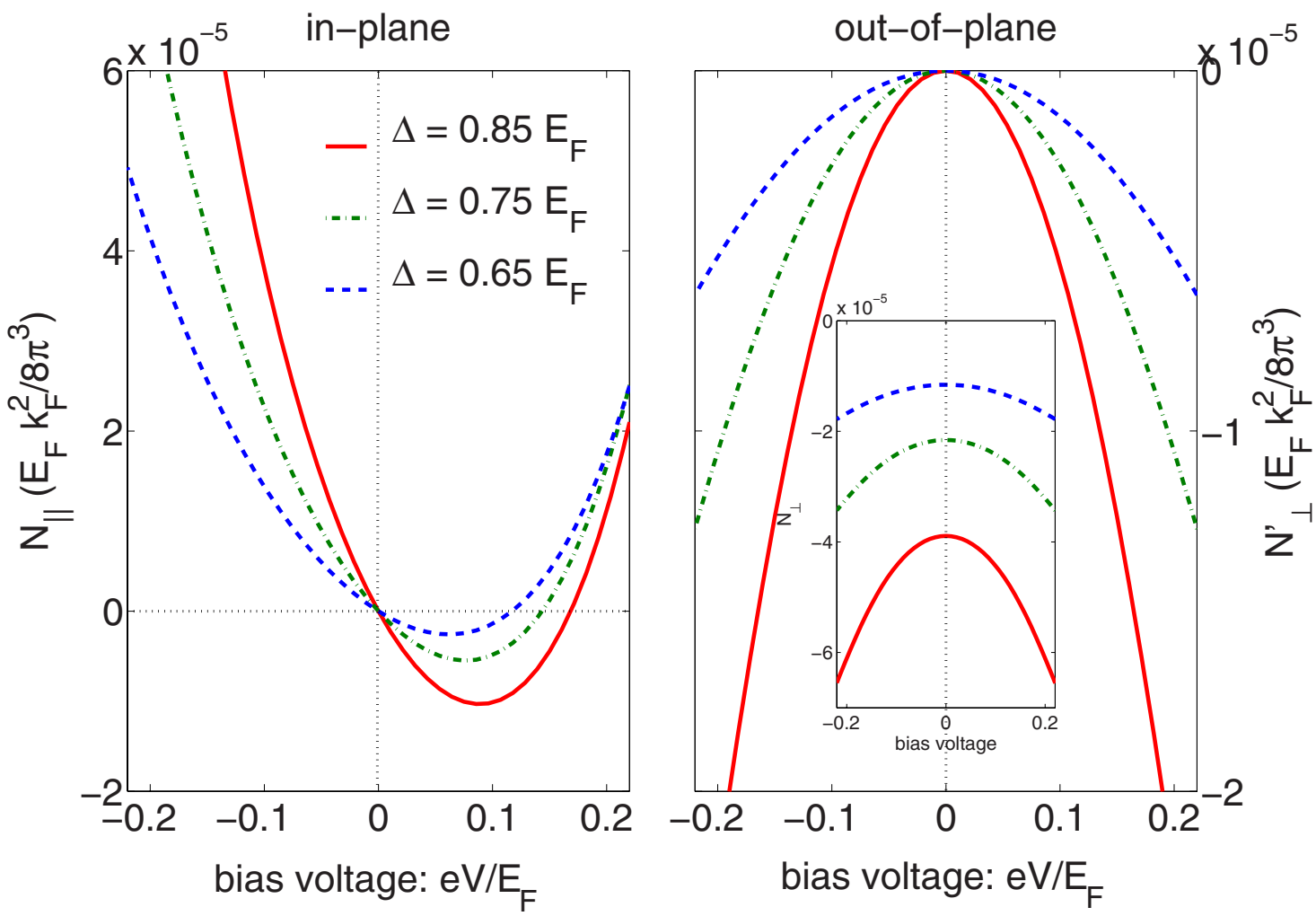

FIG. 2. (Color online) The magnitude of $\mathbf{N}_{\|}$(left) and $\mathbf{N}_{\perp}^{\prime}$ (right) acting on the right magnetization in Fig. 1 vs applied bias $e V$ for $\beta$ $=0.4$ and $d=1.0 \mathrm{~nm}$. Inset figure shows the out-of-plane torque including the equilibrium contribution at zero bias.

different exchange splitting, and (c) an FNIF structure in which a nonmagnetic layer is inserted between the insulator barrier layer and one of the ferromagnetic layers.

\section{A. Symmetric ferromagnetic-insulator-ferromagnetic}

For a symmetric Fe/MgO/Fe MTJ: $k_{F}^{\uparrow}=1.09 \AA^{-1}$ and $k_{F}^{\downarrow}$ $=0.42 \AA^{-1}$ for $\mathrm{Fe}^{8}$ and $U_{b} \simeq 1.0-1.2 \mathrm{eV}$ and $\beta=m_{b} / m$ $=0.4 \mathrm{~m}$ for $\mathrm{MgO} .4,34-36$ This implies $E_{F} \simeq 4.5 \mathrm{eV}, \Delta$ $\simeq 3.8 \mathrm{eV} \approx 0.85 E_{F}$, and $U_{b} \approx 0.25 E_{F}$. For an FIF structure $(a=0)$, both $S_{1}$ and $S_{2}$ contain only a single interface. Using the potential profile in Fig. 1(b), we have

$$
\begin{gathered}
t_{1}^{\sigma}=\frac{2 \sqrt{i k_{1}^{\sigma} \kappa(0) / \beta}}{k_{1}^{\sigma}+i \kappa(0) / \beta}, \quad t_{2}^{\sigma}=\frac{2 \sqrt{i k_{2}^{\sigma} \kappa(d) / \beta}}{k_{2}^{\sigma}+i \kappa(d) / \beta}, \\
r_{1}^{\prime \sigma}=\frac{-k_{1}^{\sigma}+i \kappa(0) / \beta}{k_{1}^{\sigma}+i \kappa(0) / \beta}, \quad r_{2}^{\sigma}=\frac{-k_{2}^{\sigma}+i \kappa(d) / \beta}{k_{2}^{\sigma}+i \kappa(d) / \beta},
\end{gathered}
$$

where $\sigma=\uparrow, \downarrow$ and $k_{1}^{\uparrow 2}+q^{2}=2 m E / \hbar^{2}, k_{1}^{\downarrow 2}+q^{2}=2 m(E-\Delta) / \hbar^{2}$. $k_{2}^{\sigma}$ are defined similarly with $E$ replaced by $E+e V$. We set $t_{1,2}^{\sigma}=0$ when $\operatorname{Im}\left(k_{1,2}^{\sigma}\right) \neq 0$.

Figure 2 shows the computed bias dependence of the inplane torque $\mathbf{N}_{\|}$(left) and the nonequilibrium part (i.e., not containing the equilibrium interlayer exchange coupling) of the out-of-plane fieldlike torque $\mathbf{N}_{\perp}^{\prime}(V)=\mathbf{N}_{\perp}(V)-\mathbf{N}_{\perp}(0)$ (right) at various exchange splittings for $m_{\mathrm{b}}=0.4 m$ at $d$ $=1.0 \mathrm{~nm}$. The equilibrium exchange coupling gives rise to an effective magnetic field in the LLG equations that we do not explicitly discuss. The main features of these curves are:
(1) the in-plane torque has both linear and parabolic contributions, and (2) the fieldlike torque is paraboliclike. These plots are very similar to the corresponding plots by Theodonis et al. ${ }^{15}$ meaning that the band-structure effects caused by the tight-binding approximation are not important. The inplane torque at negative bias and small positive bias is "normal," but changes sign at higher positive bias, where normal means that the direction of the torque in FIF is the same as the torque in metallic spin valves predicted, ${ }^{6}$ i.e., the torque curve appears in the second and fourth quadrants in the left panel of Fig. 2. When the torque curve is found in the first or third quadrant, we say that the torque is reversed. In the normal region, the positive bias (electron flow from right to left) favors the antiparallel configuration and a negative one the parallel configuration. In the reversed region, on the other hand, a current polarity that stabilizes the parallel configuration in the normal region has the opposite effect.

The zero crossing of the in-plane torque in Fig. 2 can be traced to the sign change of $T_{1}^{-}$in Eq. (11a), i.e., the sign change of the polarization of $S_{1}$ [the $F(L) / I$ interface $] p_{1}$ $=T_{1}^{-} / T_{1}^{+}<0 .{ }^{37}$ The polarization $p_{1} \propto \kappa^{2}(0)-k_{\uparrow} k_{\downarrow}$, which can take any sign depending on parameters chosen ${ }^{12}$ (see Appendix B for a more detailed discussion of this point). The vanishing torque phenomenon becomes more transparent without an effective-mass mismatch, i.e., for $\beta=1$ instead of $\beta$ $=0.4$ used in Fig. 2. The polarization vanishes when

$$
\kappa^{2}(0)-k_{\uparrow} k_{\downarrow}=0 .
$$

Since $\kappa(x=0)$ (near left interface) increases and $k_{\sigma}$ decreases with $q$, Eq. (18) is fulfilled at a certain critical value $q_{c}$. The 
(a)

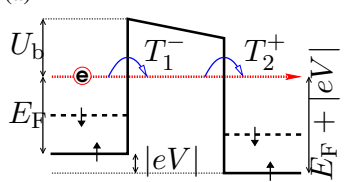

negative bias: $V<0$ (b)

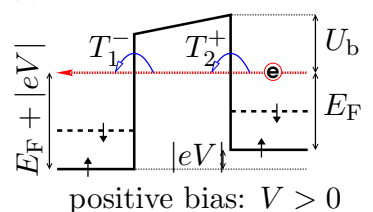

FIG. 3. (Color online) Positive and negative bias situations.

latter increases with the electron energy $E$, because $\kappa(0)$ decreases and $k_{\sigma}$ increases with $E$. This can be seen clearly from the following equation:

$$
q_{c}^{2}=\frac{2 m}{\hbar^{2}}\left[E-\frac{\left(E_{F}+U_{b}\right)^{2}}{2\left(E_{F}+U_{b}\right)-\Delta}\right] .
$$

which implies that at the Fermi energy $q_{c}$ is well defined $\left(q_{c}^{2}>0\right)$ only when $U_{b}^{2}<E_{F}\left(E_{F}-\Delta\right)$.

When $q_{c}^{2}<0$ at low bias, there is no polarization sign change for any $q$, and the (in-plane) torque behaves normally. When the potential profile becomes distorted by an applied bias as in Fig. 3(b), the electrons injected from the right lead have a maximum energy $E=E_{F}+e V$. When the applied bias $V$ is large enough, we reach the regime $q_{c}^{2}>0$, and a polarization sign change of the left interface comes into play. As $q_{c}$ increases further, more and more electron contribute to the opposite torque. When $V$ is large enough, the total torque changes sign as seen in Fig. 2. On the other hand, when the applied bias is negative [see Fig. 3(a)], the transport is dominated by the electron injected from the left lead. The electron energy and effective barrier height at the left interface do not change with applied bias, which means $T_{1}^{-}$(and so the polarization) does not change either. Therefore, we do not see a zero crossing (on the right magnetization) at negative bias in Fig. 2.

We can analogously understand the dependence of the in-plane torque on the barrier width $d$. We know that there is a polarization sign change for $q<q_{c}$ if $U_{b}$ is not too high (such that $q_{c}^{2}>0$ ). In tunneling junction, the transport is dominated by electrons with small $q$ because of the focusing effect due to the exponential extinction factor in $t_{b}$. When the electron with $q$ values smaller than $q_{c}$ dominates, the torque reverses sign. At a critical barrier width $d_{c}$, the contributions from $q<q_{c}$ cancel those from $q>q_{c}$, and the torque or torkance vanishes: $\mathbf{N}_{\|}\left(d_{c}\right)=\mathbf{T}_{\|}\left(d_{c}\right)=0$, whereas it has opposite direction for $d>d_{c}$. The left panel of Fig. 4 shows $d_{c}$ vs $U_{b}$ in linear response for various values of the barrier effective mass. $d_{c}$ increases with $U_{b}$ simply because the polarization sign change behavior is less prominent at higher barrier heights $\left(q_{c}\right.$ decreases with $\left.U_{b}\right)$.

The right panel of Fig. 4 shows the bias dependence of $d_{c}$ at barrier height $U_{b}=0.25 E_{F}$ for various barrier effective masses. Because both the positive and negative biases reduce the average barrier height, $t_{b}$ increases with $|V|$. However, the applied bias is very ineffective in changing the focusing behavior, i.e., the transmission is hardly less focused by the reduced average barrier height. This is very unlike the geometrical barrier width $d$, to which, focusing very sensitively, larger (smaller) $d$ means more (less) focusing. Rather, a posi-

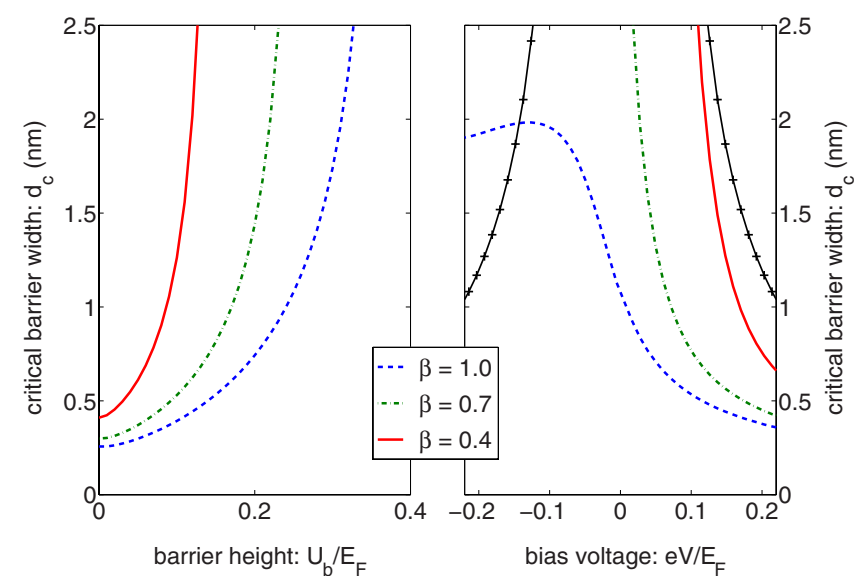

FIG. 4. (Color online) Critical barrier width $d_{c}$ vs barrier height $U_{b}$ at linear response (left) and applied bias $V$ (right). $\Delta=0.85 E_{F}$. Critical width for TMR at $\beta=0.4$ (thin solid black line with "+" symbols) is also shown in the right panel.

tive bias enhances the polarization sign change behavior, which leads to a smaller critical barrier width $d_{c}$. Since there is no polarization sign change $\left(q_{c}^{2}<0\right)$ at zero or negative bias for $\beta=0.7$ and 0.4 , a torque zero crossing is not observed. For $\beta=1.0$, polarization could change sign at zero bias, hence the torque zero crossing is also observed at negative bias. As mentioned before, negative bias does not change $T_{1}$ or polarization of the left interface, but it does change $T_{2}^{+}$. At negative bias, the barrier height at the right interface is reduced by $|e V|$, which leads to the decrease of $T_{2}^{+}$at small $q$, thus the polarization sign change behavior is weakened because of the smaller product $T_{1} T_{2}^{+}$at small $q$ where the product is negative. Weaker polarization sign changes, then requires larger $d_{c}$ for torque zero crossing at negative bias and we see $d_{c}$ increasing with negative bias for $\beta=1.0$. For comparison, a critical barrier width for the sign change of TMR is also calculated for $\beta=0.4$, and is shown as the solid black curve with "+" symbol in the right panel of Fig. 4. Since the TMR is symmetric in the applied bias for the symmetric structures, the curve is also symmetric.

\section{B. Asymmetric ferromagnetic-insulator-ferromagnetic}

As discussed at the end of Sec. III, the zero-bias (linearresponse) out-of-plane torkance does not vanish for asymmetric structures. Figure 5 shows both the in-plane and outof-plane torkances for the right FM layer at zero bias ( $V$ $=0)$ for an asymmetric FIF structure, in which the left and the right FM layers have different exchange splitting: $\Delta_{1}$ $=0.85 E_{F}$ for the left and $\Delta_{2}$ for the right, where the latter varies from 0 to $E_{F}$. From Fig. 5, we can see that the out-ofplane torkance is generally nonzero for asymmetric structures when $\Delta_{2} \neq \Delta_{1}$, and it vanishes when the right layer becomes nonmagnetic $\left(\Delta_{2}=0\right)$ or when the structure becomes symmetric $\left(\Delta_{2}=\Delta_{1}\right)$. The in-plane torkance in Fig. 5 decreases with $\Delta_{2}$ simply because the average transmission probability through the right interface $T_{2}^{+}$decreases.

\section{Ferromagnetic-normal metal-insulator-ferromagnetic}

An FNIF structure, with a nonmagnetic layer of width $a$ between one of the $F$ layers and the insulating $I$ layer, has 


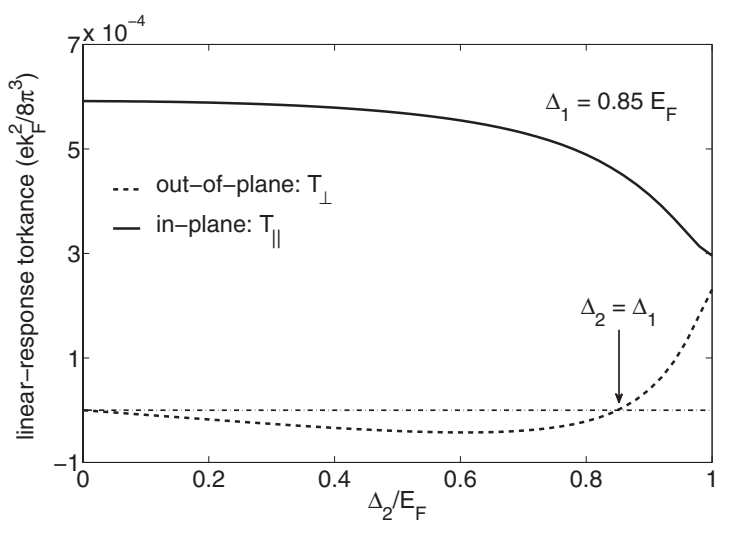

FIG. 5. Linear-response torkance vs exchange splitting $\Delta_{2}$ in the right FM $\left(\Delta_{1}=0.85 E_{F}, U_{b}=0.25 E_{F}, d=1.0 \mathrm{~nm}\right)$.

never been studied in the regime of spin-transfer torque. Such an asymmetric FNIF device can be operated in two nonequivalent modes (as in any nonsymmetric MTJ): The left $F$ is static and the right $F(\widetilde{F})$ is free (mode $1: \mathrm{FNI} \widetilde{F}$ ) and vice versa (mode 2: $\widetilde{F}$ NIF). Equations (11a)-(11c) and (12) apply to mode 1 , and apply to mode 2 with subscripts 1 and 2 exchanged. The $a$ dependence of the in-plane torkance (in linear response) is shown in Fig. 6. The sign of the in-plane torkance can be controlled by $a$ in mode 1 , but not in mode 2. This sign is determined by the sign of $T_{1}$. In mode $1, T_{1}^{-}(a)$ covers region $F(L)-N-I$ and its sign can be modulated by the $N$ insertion layer width $a$. However, in mode $2, T_{1}^{-}$covers $I-F(R)$, which is independent of $a$, therefore the sign is unchanged. The $a$ dependence of the in-plane torkance in mode 2 comes from $T_{2}^{+}(a)$, which is always positive. Due to the aliasing effect caused by discrete thickness of the $N$ layer, ${ }^{38}$ the period of the quantum oscillation in Fig. 6 should be about $\pi /\left|k_{F}-\pi / \lambda\right|$ instead of $\pi / k_{F} \approx 3 \AA$ shown in the figure, where $\lambda$ is the monolayer thickness for $N$ layer.

The asymmetry in FNIF structure shall also give rise to a finite linear-response out-of-plane torkance, which shows similar oscillations as the in-plane torkance in Fig. 6. The magnitude of the zero-bias out-of-plane torkance could be comparable to the in-plane counterpart.

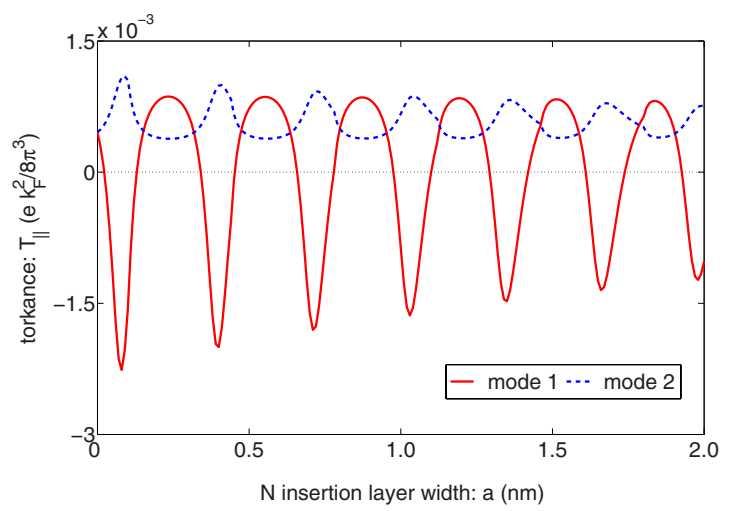

FIG. 6. (Color online) Linear-response in-plane torkance vs nonmagnetic insertion layer width $a\left(\Delta=0.85 E_{F}, U_{b}=0.25 E_{F}, d\right.$ $=1.0 \mathrm{~nm}$ ).

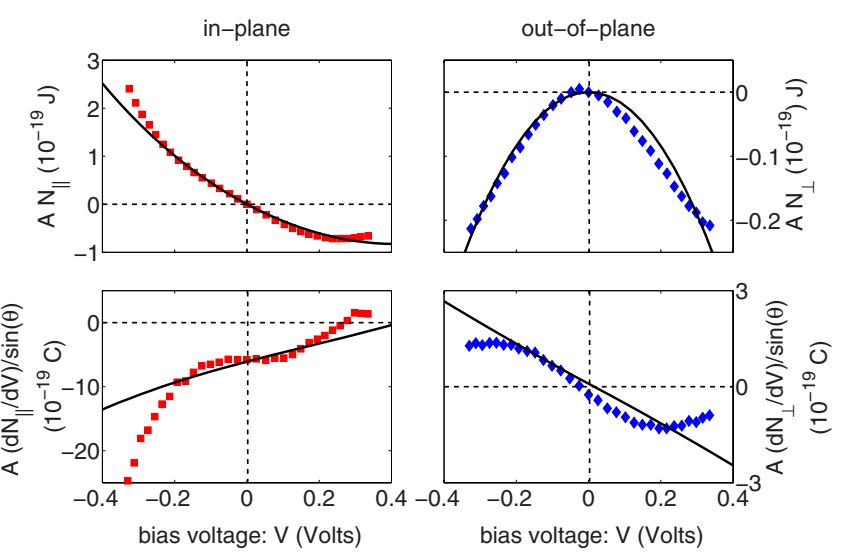

FIG. 7. (Color online) Fitting (solid curve) of the experimental data (dots) from Ref. 22. Top: in-plane torque $A N_{\|}$(left) and outof-plane torque $A N_{\perp}$ (right); Bottom: in-plane torkance $A\left(d N_{\|} / d V\right) / \sin \theta$ (left) and out-of-plane torkance $A\left(d N_{\perp}^{\prime} / d V\right) / \sin \theta$ (right). The following parameters are used in all fittings: $E_{F}$ $=4.5 \mathrm{eV}, \Delta=0.87 E_{F} \approx 3.9 \mathrm{eV}, U_{b}=0.23 E_{F} \approx 1.0 \mathrm{eV}, \beta=0.36 ; d$ $=1 \mathrm{~nm}$, cross-section area $A=70 \mathrm{~nm} \times 250 \mathrm{~nm}$ and $\theta=137^{\circ}$ from Ref. 22.

\section{COMPARISON WITH EXPERIMENTS}

Using realistic material parameters and the geometry parameters provided by Ref. 22 , we are able to reproduce, even the absolute scale, the experimental data from Ref. 22 as shown in Fig. 7, which includes the bias dependence of the in-plane torque $\mathbf{N}_{\|}(V)$ and the nonequilibrium part of the outof-plane torque $\mathbf{N}_{\perp}^{\prime}(V)=\mathbf{N}_{\perp}(V)-\mathbf{N}_{\perp}(0)$ and the corresponding torkance. The experimental data for the torkance in the bottom panels of Fig. 7 are adapted from Fig. S3(d) $\left(\beta_{S T, F T}^{\prime}\right)$ and Fig. 2 (I-V data) of Ref. 22 by $\left(d N_{\|, \perp} / d V\right) / \sin \theta$ $=(d I / d V)\left(d N_{\|, \perp} / d I\right) / \sin \theta=(d I / d V) \beta_{S T, F T}^{\prime}$. Our model appears to have a problem with the upturn of the torque at higher positive bias. The fit in Ref. 22 based on the tightbinding model of Ref. 15 is slightly better in this respect for a rather large exchange splitting. However, the out-plane torque is poorly reproduced for the same parameter set. In contrast, we succeed with a single set of (realistic) parameters to reproduce both in-plane and out-of-plane torques (torkance). The resistance for this particular device in our model is $R\left(\theta=137^{\circ}\right) \approx 150 \Omega$, which is consistent with the experimental values $(\sim 200 \Omega)$. However, the TMR value in our calculation (about 15\%) is considerably smaller than that in the experiment. We believe that the spin-transfer torque is better represented by the free-electron model than the TMR because TMR $\propto p_{1} p_{2}$, whereas torque on $\mathbf{m}_{2} \propto p_{1}$. If the polarization $p_{1}, p_{2}$ at the interfaces are underestimated by a factor of $\eta$, the TMR value is too small by a factor of $\eta^{2}$, whereas the spin-transfer torque is affected only by a factor of $\eta$ (see also Sec. VI). In addition to this, the TMR value depends sensitively on the exchange splitting $\Delta$. For instance, the TMR value increases from $15 \%$ to $30 \%$ when $\Delta$ increases from $0.87 E_{F}$ to $0.9 E_{F}$,

Another set of experimental data is shown in Fig. 8 adopted from Fig. 3(a) of Ref. 21. The experimental data are now the in-plane (red squares) and out-of-plane (blue dia- 


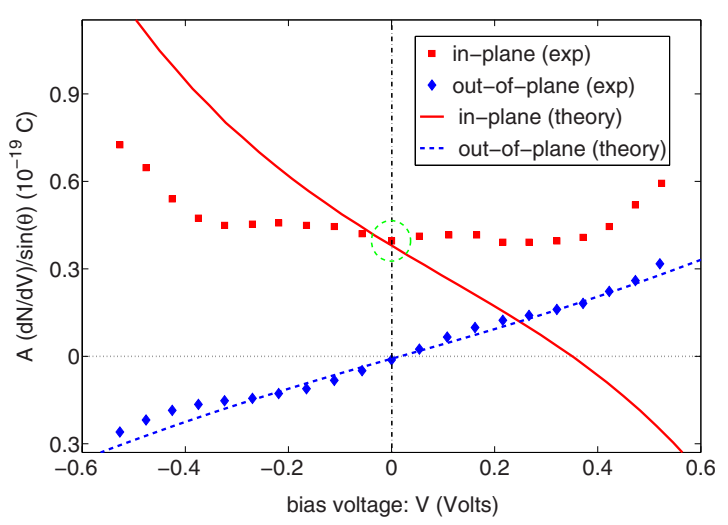

FIG. 8. (Color online) Fitting (curves) of the experimental data (dots) from Ref. 21. The following parameters are used in both fittings: $E_{F}=4.5 \mathrm{eV}, \Delta=0.85 E_{F} \approx 3.8 \mathrm{eV}, U_{b}=0.25 E_{F} \approx 1.1 \mathrm{eV}$, $\beta=0.43 ; d=1.25 \mathrm{~nm}$, cross-section area $A=50 \mathrm{~nm} \times 100 \mathrm{~nm}$, and $\theta=71^{\circ}$ from Ref. 21 (notice the sign convention in Ref. 21 is opposite to that in Ref. 22).

monds) torkance. We fit the out-of-plane and the linear part of the in-plane torkance again on absolute scale. Theory contains a quadratic component of the in-plane torque, which does not show up in this experiment. The resistance for this particular device in our model is $R\left(\theta=71^{\circ}\right) \approx 4 \mathrm{k} \Omega$, consistent with the experimental value $(\sim 3.5 \mathrm{k} \Omega)$, whereas our TMR, 5\%, again is too small. Note that the torkances are much smaller than in Ref. 22 because of the thicker barrier and the smaller cross-section area, as reflected by the higher resistance.

Deac et al. ${ }^{23}$ also measured the in-plane and out-of-plane torque in a $\mathrm{MgO}$ based tunneling junction. The out-of-plane torque in this experiment agrees well with other experiments and theory. However, the in-plane torque depends parabolically on the applied bias ( $A V^{2}$ for positive bias and $-B V^{2}$ for negative bias, where $A$ and $B$ are positive constants), which is quite different from both Refs. 21 and 22. A voltage noise measurements done by Petit et al. ${ }^{16}$ also suggest a linear out-of-plane torkance (or parabolic out-of-plane torque), which is about $20 \%$ of the in-plane counterpart. Hence all experiments and theories appear to agree on the out-of-plane torque, whereas consensus about the in-plane torque has not been reached yet.

\section{DISCUSSION}

Because of the high quality of epitaxial $\mathrm{MgO}$ tunnel layers, ${ }^{4,5}$ we ignored interface roughness and barrier disorder. The main effect of the geometric interface roughness is to reduce the nominal thickness of the barrier. ${ }^{39}$ Impurity states in the barrier generally increase tunneling because of the opening of additional tunneling channels with lower barrier height $U_{b}^{\prime}<U_{b}$. Impurities states also weaken the spindependent effects when spin flip is involved. In general, interface roughness and disorder can be important quantitatively, but have been shown not to qualitatively change the features predicted by a ballistic model. ${ }^{40-43}$

The free-electron Stoner model is only a poor representation of the real electronic structure of transition metals for the tunneling problem: It fails to properly reproduce the nearly half-metallic nature of transition-metal ferromagnets based $\mathrm{MgO}$ tunnel junctions, that is caused by the symmetry of the bands at the Fermi energy, ${ }^{44}$ leading to the underestimated TMR ratios by our model noted above. On the other hand, the band-structure calculations by Heiliger and Stiles ${ }^{17}$ show that the free-electron model can perform quite well as far as the torque is concerned. We explained this in Sec. IV by its dependence on only one interface polarization leading to a better performance of a model that is not accurate in this respect. The band-structure calculations in Ref. 17 indicate that the torque is strongly localized to a few monolayers which is in support of our simple model.

The issue of the wave-function symmetry should also be considered when a normal metal is inserted. When the electrons with wave vector normal to the interface dominate, the normal metal $(\mathrm{Cr})$ is actually a potential barrier for the $\mathrm{Fe}$ majority spins ${ }^{45}$ rather than a potential well as assumed here.

In contrast to metallic spin valve structures, in which the out-of-plane torque is generally less than $10 \%$ of the in-plane counterpart, the out-of-plane contribution has been found quite large in tunneling junctions (a 30\% contribution at high bias is measured in Ref. 21). Close to the zero crossing of the in-plane torque at positive bias the out-of-pane torque should become dominant.

An experimental "phase diagram" that can be compared with Fig. 4 would constitute a stringent test of our predictions. Since the barrier height and the effective electron mass in the barrier cannot be controlled, we suggest measuring the torques systematically for several MTJ structures with different barrier width (otherwise identical) to test the red curve in the right panel of Fig. 4. The zero crossing of the in-plane torque is predicted to occur at voltages that are too high for the experiments in Refs. 21-23. For wider tunneling barriers it should happen at smaller voltages.

\section{SUMMARY}

To summarize, scattering theory of transport is used to calculate the spin-transfer torque for a magnetic multilayer structure at finite bias. The experimental spin-torque data (both in-plane and out-of-plane) can be reproduced using realistic parameters in our model. The spin-transfer torque on a given layer may change sign for only one bias polarity. The torque zero crossing is caused by the combined effects of the polarization sign change at the FI interface and the focusing effect of the barrier. The bias voltage required for torque zero-crossing decreases as a function of the barrier width. The out-of-plane torkance at zero bias vanishes for symmetric FIF structures, but remains finite for asymmetric structures. In FNIF structure we find on top of the previously reported oscillating TMR (Ref. 3) and charge pumping voltage $^{33}$ that the spin-transfer torque also oscillates and may change sign with the $N$ layer thickness.

\section{ACKNOWLEDGMENTS}

We acknowledge M. D. Stiles, C. Heiliger, and A. Deac 


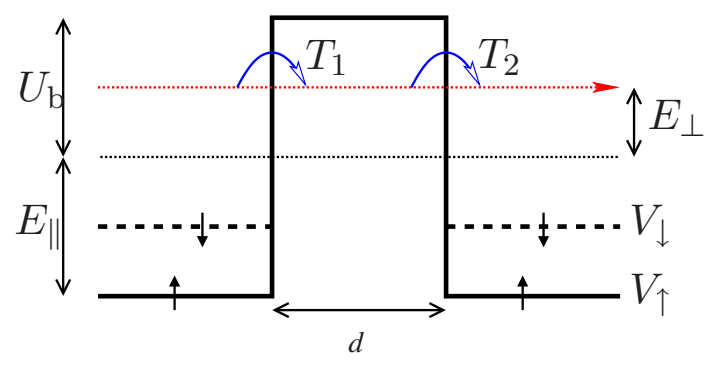

FIG. 9. (Color online) Potential barrier.

for helpful discussions. This work is supported by EC Contract No. IST-033749 "DynaMax."

\section{APPENDIX A: OPTICAL THEOREM IN TUNNEL JUNCTION}

The scattering matrices in Eq. (2a) and (2b) include the reflection amplitude inside the insulator $\left(\hat{r}_{1}^{\prime}\right.$ and $\left.\hat{r}_{2}\right)$. Expressions become more transparent when the reflection amplitudes are replaced by the transmission probabilities, however. This can be achieved by the optical theorem tailored for tunnel junction that reflects current conservation. Note that the equivalent statement in metallic systems is the wellknown relation $|r|^{2}+|t|^{2}=1$ that follows from the unitary of the scattering matrix.

The optical theorem for light is derived from conservation of energy, whereas in electronic transport it is based on conservation of charge. We consider here the nonstandard situation of the interface between a metal and a tunneling barrier, for which the unitary of the scattering matrix cannot be invoked without some care. Let us consider a nonmagnetic $I X N$ structure, where $X$ could be basically anything. We assume flux-normalized plane waves $e^{i k_{n} x} / \sqrt{k_{n}}$ in $N$ with mode index $n$, and exponential solutions $e^{ \pm \kappa_{m} x} / \sqrt{\kappa_{m}}$ in $I$ with mode index $m$. The electron with wave function $e^{-\kappa_{p} x} / \sqrt{\kappa_{p}}$ in $I$ is reflected with amplitude $r_{m p}$, and transmitted into $N$ with amplitude $t_{n p}$. Then the wave functions in $I$ and $N$ are:

$$
\psi_{I}^{m}=\frac{e^{-\kappa_{p} x}}{\sqrt{\kappa_{p}}} \delta_{m p}+r_{m p} \frac{e^{\kappa_{m} x}}{\sqrt{\kappa_{m}}}, \quad \psi_{N}^{n}=t_{n p} \frac{e^{i k_{n} x}}{\sqrt{k_{n}}} .
$$

The current in $I$ is given by the imaginary part of the reflection amplitudes since

$$
I_{I}=\frac{\hbar}{m} \sum_{m} \operatorname{Im}\left(\psi_{\mathrm{I}}^{m *} \partial_{x} \psi_{I}^{m}\right)=\frac{2 \hbar}{m} \operatorname{Im}\left(r_{p p}\right),
$$

The current in $N$ reads

$$
I_{N}=\frac{\hbar}{m} \sum_{n} \operatorname{Im}\left(\psi_{N}^{n *} \partial_{x} \psi_{N}^{n}\right)=\frac{\hbar}{m} \sum_{n}\left|t_{n p}\right|^{2} .
$$

By current conservation: $I_{I}=I_{N}$, we have

$$
2 \operatorname{Im}\left(r_{p p}\right)=\sum_{n}\left|t_{n p}\right|^{2} \equiv \sum_{n} T_{n p}
$$

This relation reduces to

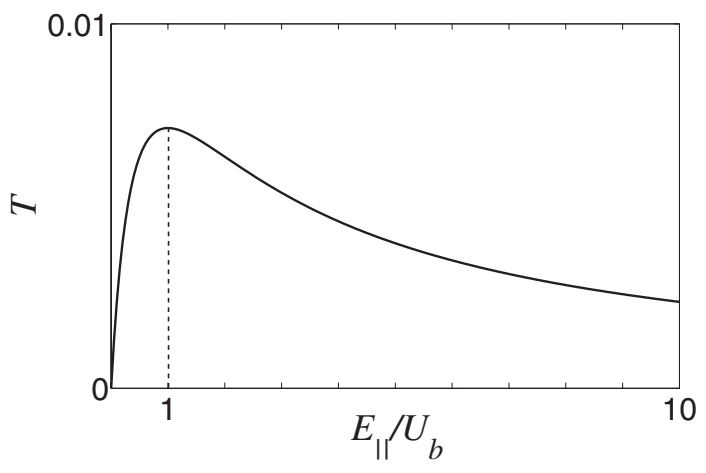

FIG. $10 . T$ vs. $E_{\|} / U_{b}\left(U_{b}=0.25 E_{F}, E=E_{F}, \beta=0.4\right.$, and $d$ $=1 \mathrm{~nm})$.

$$
2 \operatorname{Im}\left(r_{p p}\right)=\left|t_{p p}\right|^{2} \equiv T_{p p},
$$

for the ballistic model used in the text.

\section{APPENDIX B: POLARIZATION SIGN CHANGE}

For a better understanding of the polarization sign change, let us inspect the simple potential barrier depicted in Fig. 9 (thick solid line), ignoring the spin dependence for the moment. The barrier width is $d$ and the relative barrier height $U_{b}=\hbar^{2} \kappa^{2} / 2 m$. As seen in Fig. 9, $U_{b}$ is measured relative to the longitudinal electron energy in the barrier $E-E_{\perp}=E_{\|}$ $+V$, where $E$ is the total electron energy, $E_{\|}$and $E_{\perp}$ are the longitudinal (normal to the interfaces) and transverse kinetic energies, and $V$ is the band edge. By solving this standard quantum-mechanical exercise, we find the transmission probability through the barrier

$$
T=\frac{1}{1+\frac{\left(E_{\|}+U_{b}\right)^{2}}{4 E_{\|} U_{b}} \sinh ^{2}(\kappa d)} \approx \frac{16 E_{\|} U_{b}}{\left(E_{\|}+U_{b}\right)^{2}} e^{-2 \kappa d},
$$

where the approximation is accurate when $\kappa d \gg 1$. Equation (B1) shows that for a fixed $U_{b}$ (or $\left.\kappa\right), T$ is maximal when $E_{\|} / U_{b}=1$ (see Fig. 10 ), where $E_{\|}$can be tuned by changing the band edge $V$.

In an FIF MTJ, for electrons in $F$ with the same total energy $E=E_{\|}^{\uparrow / \downarrow}+V_{\uparrow / \downarrow}+E_{\perp}$ and the same transverse energy $E_{\perp}$, the relative barrier height $\left[U_{b}\right.$ in Eq. (B1)] is the same for spin-up and spin-down electrons. However, the band edges $\left(V_{\uparrow / \downarrow}\right)$ are spin dependent as indicated by the solid (spin up) and dashed (spin down) lines in Fig. 9. The longitudinal kinetic energies $\left[E_{\|}\right.$in Eq. (B1) $]$is larger for spin-up than spin-down electrons. In our Stoner model we typically find $E_{\|}^{\downarrow} / U_{b} \sim 1$, whereas $E_{\|}^{\uparrow} / U_{b}>2$. According to Eq. (B1) and Fig. 10, we find for the parameters in our Stoner model the surprising result that $T_{\uparrow}<T_{\downarrow}$. In general, electrons close to the Fermi energy with small transverse wave vectors $q$ show this inverted polarization sign change behavior. For large $q$, spin-up electrons tend to have higher transmission again, and the polarization becomes positive. 
${ }^{1}$ X. G. Zhang and W. H. Butler, J. Phys.: Condens. Matter 15, R1603 (2003).

${ }^{2}$ E. Y. Tsymbal, O. N. Mryasov, and P. R. LeClair, J. Phys.: Condens. Matter 15, R109 (2003).

${ }^{3}$ S. Yuasa, T. Nagahama, and Y. Suzuki, Science 297, 234 (2002).

${ }^{4}$ S. Yuasa, T. Nagahama, A. Fukushima, Y. Suzuki, and K. Ando, Nat. Mater. 3, 868 (2004)

${ }^{5}$ S. S. P. Parkin, C. Kaiser, A. Panchula, P. M. Rice, B. Hughes, M. Samant, and S.-H. Yang, Nat. Mater. 3, 862 (2004).

${ }^{6}$ J. C. Slonczewski, J. Magn. Magn. Mater. 159, L1 (1996).

${ }^{7}$ L. Berger, Phys. Rev. B 54, 9353 (1996).

8 J. C. Slonczewski, Phys. Rev. B 39, 6995 (1989).

${ }^{9}$ T. Miyazaki and N. Tezuka, J. Magn. Magn. Mater. 139, L231 (1995).

${ }^{10}$ J. S. Moodera, L. R. Kinder, T. M. Wong, and R. Meservey, Phys. Rev. Lett. 74, 3273 (1995).

${ }^{11}$ Y. Huai, F. Albert, P. Nguyen, M. Pakala, and T. Valet, Appl. Phys. Lett. 84, 3118 (2004).

${ }^{12}$ J. C. Slonczewski, Phys. Rev. B 71, 024411 (2005).

${ }^{13}$ A. Kalitsov, I. Theodonis, N. Kioussis, M. Chshiev, W. H. Butler, and A. Vedyayev, J. Appl. Phys. 99, 08 G501 (2006).

${ }^{14}$ P. M. Levy and A. Fert, Phys. Rev. Lett. 97, 097205 (2006).

${ }^{15}$ I. Theodonis, N. Kioussis, A. Kalitsov, M. Chshiev, and W. H. Butler, Phys. Rev. Lett. 97, 237205 (2006).

${ }^{16}$ S. Petit, C. Baraduc, C. Thirion, U. Ebels, Y. Liu, M. Li, P. Wang, and B. Dieny, Phys. Rev. Lett. 98, 077203 (2007).

${ }^{17}$ C. Heiliger and M. D. Stiles, Phys. Rev. Lett. 100, 186805 (2008).

${ }^{18}$ A. Manchon, N. Ryzhanova, A. Vedyayev, M. Chschiev, and B. Dieny, J. Phys.: Condens. Matter 20, 145208 (2008).

${ }^{19}$ M. Wilczynski, J. Barnas, and R. Swirkowicz, Phys. Rev. B 77, 054434 (2008).

${ }^{20}$ A. Manchon, N. Ryzhanova, M. Chschiev, A. Vedyayev, K.-J. Lee, and B. Dieny, arXiv:0802.3754 (unpublished).

${ }^{21}$ J. C. Sankey, Y. T. Cui, R. A. Buhrman, D. C. Ralph, J. Z. Sun, and J. C. Slonczewski, Nat. Phys. 4, 67 (2008).

${ }^{22}$ H. Kubota et al., Nat. Phys. 4, 37 (2008).

${ }^{23}$ A. M. Deac, A. Fukushima, H. Kubota, H. Maehara, Y. Suzuki,
S. Yuasa, Y. Nagamine, K. Tsunekawa, D. D. Djayaprawira, and W. Naoki, arXiv:0803.2013 (unpublished).

${ }^{24}$ J. Z. Sun and D. C. Ralph, J. Magn. Magn. Mater. 320, 1227 (2008).

${ }^{25}$ A. Brataas, Y. V. Nazarov, and G. E. W. Bauer, Phys. Rev. Lett. 84, 2481 (2000).

${ }^{26}$ X. Waintal, E. B. Myers, P. W. Brouwer, and D. C. Ralph, Phys. Rev. B 62, 12317 (2000).

${ }^{27}$ J. C. Slonczewski, J. Magn. Magn. Mater. 247, 324 (2002).

${ }^{28}$ M. D. Stiles and A. Zangwill, Phys. Rev. B 66, 014407 (2002).

${ }^{29}$ J. Xiao, A. Zangwill, and M. D. Stiles, Eur. Phys. J. B 59, 415 (2007).

${ }^{30}$ J. C. Slonczewski and J. Z. Sun, J. Magn. Magn. Mater. 310, 169 (2007).

${ }^{31}$ Y. Tserkovnyak, A. Brataas, G. E. W. Bauer, and B. I. Halperin, Rev. Mod. Phys. 77, 1375 (2005).

32 J. C. Slonczewski, J. Magn. Magn. Mater. 126, 374 (1993).

${ }^{33}$ J. Xiao, G. E. W. Bauer, and A. Brataas, Phys. Rev. B 77, 180407(R) (2008).

${ }^{34}$ J. S. Moodera and L. R. Kinder, J. Appl. Phys. 79, 4724 (1996).

${ }^{35}$ M. Bowen et al., Appl. Phys. Lett. 79, 1655 (2001).

${ }^{36}$ J. Faure-Vincent, C. Tiusan, C. Bellouard, E. Popova, M. Hehn, F. Montaigne, and A. Schuhl, Phys. Rev. Lett. 89, 107206 (2002).

${ }^{37}$ F. F. Li, Z. Z. Li, M. W. Xiao, J. Du, W. Xu, A. Hu, and J. Q. Xiao, J. Appl. Phys. 95, 7243 (2004)

${ }^{38}$ C. Chappert and J. P. Renard, Europhys. Lett. 15, 553 (1991).

${ }^{39}$ S. Zhang and P. M. Levy, Eur. Phys. J. B 10, 599 (1999).

${ }^{40}$ H. Itoh, A. Shibata, T. Kumazaki, J. Inoue, and S. Maekawa, J. Phys. Soc. Jpn. 68, 1632 (1999).

${ }^{41}$ J. Mathon and A. Umerski, Phys. Rev. B 60, 1117 (1999).

${ }^{42}$ H. Itoh, J. Inoue, A. Umerski, and J. Mathon, Phys. Rev. B 68, 174421 (2003)

${ }^{43}$ P. X. Xu, V. M. Karpan, K. Xia, M. Zwierzycki, I. Marushchenko, and P. J. Kelly, Phys. Rev. B 73, 180402(R) (2006).

${ }^{44}$ X. G. Zhang and W. H. Butler, Phys. Rev. B 70, 172407 (2004).

${ }^{45} \mathrm{~S}$. Yuasa (unpublished). 